\section{References}

1. Liesveld J. Management of AML: who do we really cure? Leuk Res. 2012;36(12):1475-1480.

2. Dohner H, Estey E, Grimwade D, et al. Diagnosis and management of AML in adults: 2017 ELN recommendations from an international expert panel. Blood. 2017;129(4):424-447.

3. Thomas D, Majeti R. Biology and relevance of human acute myeloid leukemia stem cells. Blood. 2017;129(12):1577-1585

4. Lapidot T, Sirard C, Vormoor J, et al. A cell initiating human acute myeloid leukaemia after transplantation into SCID mice. Nature. 1994;367(6464):645-648.

5. Shlush LI, Zandi S, Mitchell A, et al. Identification of pre-leukaemic haematopoietic stem cells in acute leukaemia. Nature. 2014;506(7488):328-333

6. Pollyea DA, Gutman JA, Gore L, Smith CA, Jordan CT. Targeting acute myeloid leukemia stem cells: a review and principles for the development of clinical trials. Haematologica. 2014;99(8):1277-1284.

7. Ng SW, Mitchell A, Kennedy JA, et al. A 17-gene stemness score for rapid determination of risk in acute leukaemia. Nature. 2016;540(7633):433-437

8. Bill M, Nicolet D, Kohlschmidt J, et al. Mutations associated with a 17-gene leukemia stem cell score and the score's prognostic relevance in the context of the European LeukemiaNet classification for acute myeloid leukemia. Haematologica.2020;105(3):721-729.

9. Duployez N, Marceau-Renaut A, Villenet C, et al. The stem cellassociated gene expression signature allows risk stratification in pediatric acute myeloid leukemia. Leukemia. 2019;33(2):348-357.

10. DiNardo CD, Pratz K, Pullarkat V, et al. Venetoclax combined with decitabine or azacitidine in treatment-naive, elderly patients with acute myeloid leukemia. Blood. 2019;133(1):7-17.

11. Pollyea DA, Stevens BM, Jones CL, et al. Venetoclax with azacitidine disrupts energy metabolism and targets leukemia stem cells in patients with acute myeloid leukemia. Nat Med. 2018;24(12):18591866.

12. Perl AE, Martinelli G, Cortes JE, et al. Gilteritinib or chemotherapy for relapsed or refractory FLT3-mutated AML. N Engl J Med. 2019;381(18):1728-1740.

\title{
Thrombopoietin receptor agonists for the treatment of inherited thrombocytopenia
}

\author{
Michael Makris ${ }^{1,2}$ \\ ${ }^{1}$ Department of Infection, Immunity and Cardiovascular disease, University of Sheffield, and ${ }^{2}$ Sheffield Haemophilia and Thrombosis \\ Centre, Royal Hallamshire Hospital, Sheffield, UK \\ E-mail:m.makris@sheffield.ac.uk
}

doi:10.3324/haematol.2019.241786

T he inherited thrombocytopenias are a heterogeneous group of increasingly recognized disorders, which can be associated with bleeding of variable severity. Their prevalence has been estimated to be around 1 in 100,000 of the population, ${ }^{1}$ but it is likely that this is an underestimate due to many individuals being undiagnosed, wrongly diagnosed or not recorded on registries after a correct diagnosis. More recently, it has been reported that the prevalence of MYH9-related disorders can be as frequent as 1 in 20,000 of the population. ${ }^{2}$

The inherited nature of the thrombocytopenias has been recognized for decades, with the main disorders being the May-Hegglin anomaly, and the Sebastien, Fechtner and Epstein syndromes. These disorders were associated with a variable degree of renal impairment, deafness and cataracts. Although initially believed to be different disorders, when the genes responsible were identified, it became clear that all of these syndromes were variants of defects in the same $\mathrm{MYH} 9$ gene encoding for non-muscle myosin heavy chain $\mathrm{A} .{ }^{3}$ The nomenclature was subsequently changed to reflect this, and they are now known as the MYH9-related disorders (MYH9-RD).

The recent introduction of high throughput sequencing (HTS), together with the formation of consortia with large numbers of clinicians caring for inherited thrombocytopenia patients, has led to a dramatic increase in the number of genes responsible for the disorder. Inherited thrombocytopenias can be syndromic, predisposing to renal failure, hearing loss and cataracts, as in MYH9-RD, while others, such as the RUNX1, ANKRD26 and ETV6, can be associated with predisposition to hematologic malignancy, 4,5

In contrast to the major advances in the genetic basis of inherited thrombocytopenia, the management of these disorders has hardly changed, with the main therapeutic decision being whether to transfuse platelets or not. Part of the difficulty is the variability in the number of platelets, as well as the bleeding tendency which is often not directly proportional to the platelet count. A possible explanation for this is the variable and often large size of the platelets in some of these disorders; since hemostatic reactions take place on the cell surface, disorders associated with larger platelets would be expected to be associated with less bleeding. Treatment is usually required when patients are actively bleeding, or to prevent bleeding prior to surgery or invasive procedures.

Platelet transfusions, however, can be problematic because of the potential for adverse events. They carry the risk of transfusion-transmitted infection, alloimmunization with production of platelet specific or HLA antibodies, allergic reactions and transfusion-related acute lung injury (TRALI). As a result, the use of platelet transfusions tends to be avoided if possible, and clinicians use tranexamic acid, sometimes with desmopressin, as nonspecific hemostatic agents to treat these patients.

Thrombopoietin receptor agonists have been available for the treatment of immune thrombocytopenia in adults and children for some time. The two products with the longest availability are eltrombopag, which is given orally, and romiplostim, which is administered subcutaneously. In the UK, eltrombopag is available for use in patients with thrombocytopenia of at least six months duration whilst romiplostim is approved for ITP of 12 months duration or more.

In an important initial publication from 2010, Pecci et al. showed that eltrombopag could increase the platelet count of patients with $M Y H 9$-related thrombocytopenia. ${ }^{\circ}$ Twelve patients with a platelet count of $<50 \times 10^{\circ} / \mathrm{L}$ were 
treated with 50mg eltrombopag for three weeks, and those who did not achieve a platelet count of $>100 \times 10^{9} / \mathrm{L}$ received an additional three weeks of treatment at $75 \mathrm{mg}$ once daily. Five patients achieved a platelet count of $>100 \times 10^{9} / \mathrm{L}$ with the $50 \mathrm{mg}$ dose and an additional three achieved this with the $75 \mathrm{mg}$ dose. Overall, $67 \%$ of patients achieved a platelet count of $>100 \times 10 \% / \mathrm{L}, 25 \%$ achieved a minor response, and a single patient did not respond. The treatment was well tolerated, and the bleeding symptoms resolved in 8 of 10 patients who presented these at study entry. ${ }^{6}$

Since it is known that in most forms of inherited thrombocytopenia the megakaryocytes respond to TPO receptor agonists, the next logical step was to investigate these agents for the treatment of other forms of inherited thrombocytopenia, and this is what Zaninetti et al. have done. ${ }^{8}$ In this issue of the Journal, they report on their multicenter prospective investigation of eltrombopag in patients with five different types of inherited thrombocytopenia. A total of 24 patients with $M Y H 9$-related disease, ANKRD26-related thrombocytopenia, X-
linked/Wiskott-Aldrich syndrome, monoallelic BernardSoullier syndrome, and ITGB3-related thrombocytopenia were included. Patients awaiting procedures received an escalating dose of $50-75 \mathrm{mg}$ eltrombopag for 3-6 weeks, while individuals with active bleeding received an escalating dose of $25-75 \mathrm{mg}$ for up to 16 weeks (Figure 1). The responses varied between the different defects, but overall $48 \%$ of the patients responded, achieving a platelet count of over $100 \times 10^{9} / \mathrm{L}$. All four patients who were experiencing mucosal bleeding on entry, stopped bleeding following the eltrombopag treatment. The treatment with eltrombopag was well tolerated, but one patient with Wiskott Aldrich syndrome discontinued the treatment due to deterioration of his eczema.

The trial by Zanetti et al. excluded some patients with inherited thrombocytopenia and predisposition to hematologic malignancy, such as those with mutations in the RUNX1 (previously known as AML1) and ETV6 genes; $A N K R D 26$ patients were, however, included. The evidence that TPO receptor agonists accelerate disease progression is controversial. A study of the use of the TPO

\section{Inherited thrombocytopenia}

? Active bleeding symptoms

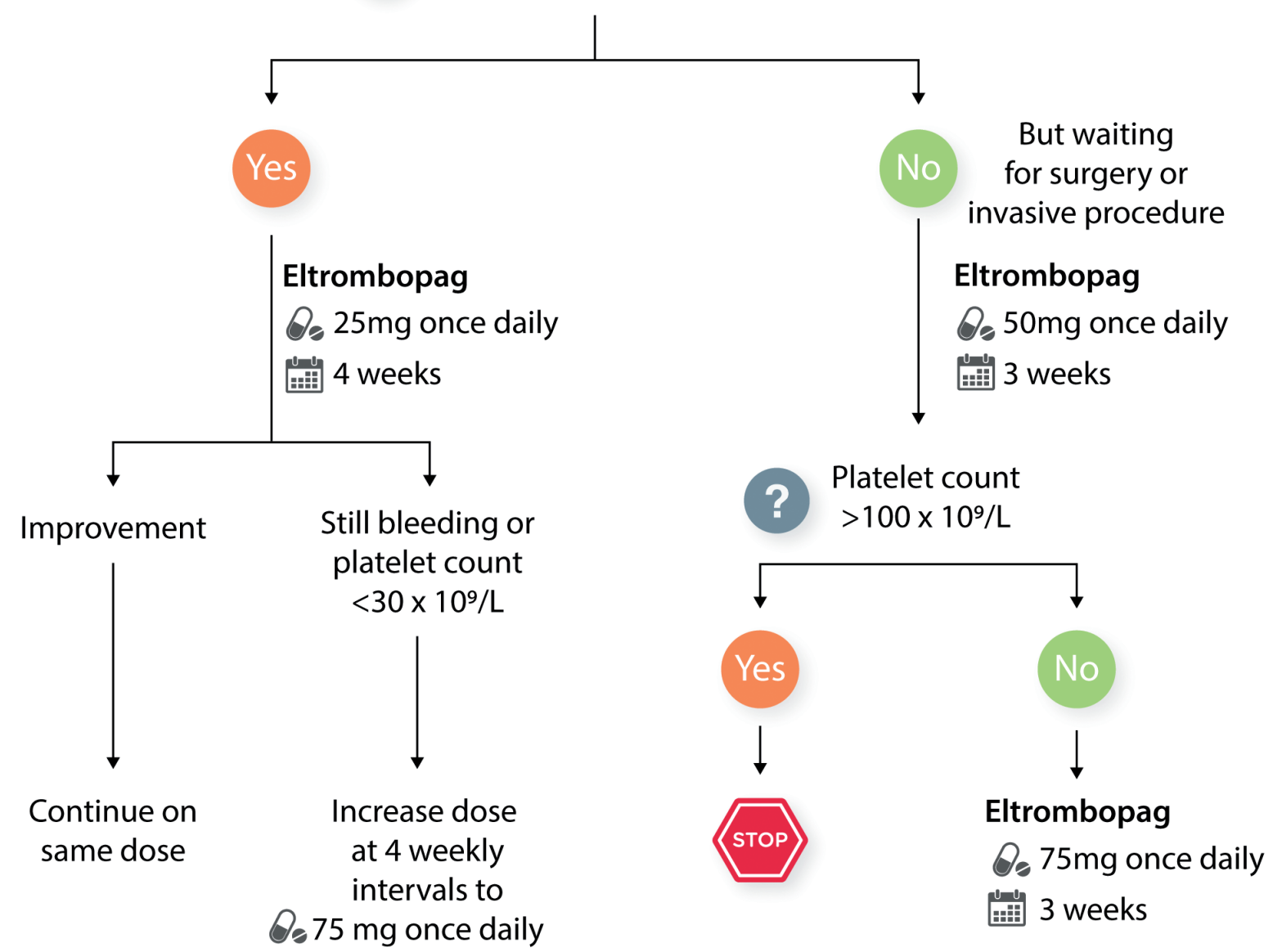

Figure 1. Outline of the phase II clinical trial using eltrombopag in patients with inherited thrombocytopenia. 
receptor agonist romiplostim in patients with myelodysplasia and thrombocytopenia was halted early despite leading to an increase in the platelet count and improvement in bleeding symptoms because of concern regarding progression to acute myeloid leukemia (AML). In the final analysis, however, the AML progression risk was not significant, with a hazard ratio of 1.20 and a $95 \%$ confidence interval of $0.38-3.84 .^{9}$ No such concern was observed in a subsequent trial of eltrombopag monotherapy in similar settings. ${ }^{10}$

As mentioned before, there are at least 40 different genes associated with inherited thrombocytopenia. ${ }^{11}$ At this stage, it is not known whether patients with other gene mutations will respond to eltrombopag in the same way, or whether any of these disorders will respond to the other TPO receptor agonists such as romiplostim; while the answer to both of these questions is likely to be yes, this is still speculation and needs to be confirmed in clinical trials or case series.

Considering the rarity and variety of the inherited thrombocytopenias, as well as the brief period for which most of these individuals require treatment to improve their platelet count, it is unlikely that a pharmaceutical manufacturer will perform the required trials to get approval of their drug for this indication.

The study by Zanetti et al. is important because it establishes eltrombopag as a therapeutic entity in the treatment of inherited thrombocytopenia. At this stage, we do not know if patients with mutations in other genes, or even different mutations in the same gene, will respond the same way, if at all. In view of this, it would be sensible to offer patients soon after diagnosis a 3-week therapeutic trial of $50 \mathrm{mg}$ of eltrombopag daily with the option of another three weeks at 75mg daily in the nonor poor-responders. In this way, at moments of possible future need, it will already be known whether they are eltrombopag responders or not, in which case they are likely to require platelet transfusions. I believe that, for elective procedures, the use of eltrombopag as a first-line agent is a very reasonable proposition, even when the drug is not licensed for this indication.
Although in many countries there are national registries of patients with inherited bleeding disorders, these tend to be for individuals with clotting factor deficiencies and do not include persons with inherited thrombocytopenia. I believe that all patients with inherited thrombocytopenia should be entered in registries so that the natural history, as well as the response to TPO receptor agonist treatment for all the different genetic defects, can be established. It is unlikely that this will be achieved for most of the disorders without international collaboration.

\section{References}

1. Balduini CL, Pecci A, Noris P. Diagnosis and management of inherited thrombocytopenias. Semin Thromb Hemost. 2013;39(2):161-171.

2. Fernandez-Prado R, Carriazo-Julio SM, Torra R, Ortiz A, PerezGomez MV. MYH9-related disease: it does exist, may be more frequent than you think and requires specific therapy. Clin Kidney J. 2019;12(4):488-493.

3. Kelley MJ, Jawien W, Ortel TL, Korczak JF. Mutation of MYH9, encoding for non-muscle myosin heavy chain A, in May-Hegglin anomaly. Nat Genet. 2000;26(1):106-108.

4. Noris P, Pecci A. Hereditary thrombocytopenias: a growing list of disorders. Hematology Am Soc Hematol Educ Program. 2017;2017(1):385-399.

5. Galera P, Dulau-Florea A, Calvo KR. Inherited thrombocytopenia and platelet disorders with germline predisposition to myeloid neoplasia. Int J Lab Hematol. 2019;41 Suppl 1:131-141.

6. Pecci A, Gresele P, Klersy C, et al. Eltrombopag for the treatment of the inherited thrombocytopenia deriving from MYH9 mutations. Blood. 2010;116(26):5832-5837.

7. Pecci A. Pathogenesis and management of inherited thrombocytopenias: rationale for the use of thrombopoietin-receptor agonists. Int J Hematol. 2013;98(1):34-47.

8. Zaninetti C, Gresele P, Bertomoro A, et al. Eltrombopag for the treatment of inherited thrombocytopenias: a phase 2 clinical trial. Haematologica. 2020;105(3):820-828.

9. Giagounidis A, Mufti GJ, Fenaux P, et al. Results of a randomized double-blind study of romiplostim versus placebo in patients with low/intermediate-1-risk myelodysplastic syndrome and thrombocytopenia. Cancer. 2014;120(12):1838-1846.

10. Mittelman M, Platzbecker U, Afanasyev B, et al. Eltrombopag for advanced myelodysplastic syndromes or acute myeloid leukaemia and severe thrombocytopenia (ASPIRE): a randomised, placebo-controlled, phase 2 trial. Lancet Haematol. 2018;5(1):e34-e43.

11. Almazni I, Stapley R, Morgan NV. Inherited Thrombocytopenia: Update on genes and genetic variants which may be associated with bleeding. Front Cardiovasc Med. 2019;6:80. 